\title{
Implementing of High Capacity Tbps DWDM System Optical Network
}

\author{
Daleep Singh Sekhon*, Harmandar Kaur \\ Deptt.of ECE, GNDU Regional Campus, Jalandhar,Punjab,India \\ *dssekhon24@gmail.com
}

\begin{abstract}
In this paper we investigate the high speed Tbps (Terabits per second) DWDM (Dense Wavelength Division Multiplexing) system optical network. In this system there is present 40 channels at transmitter section each having bit rate of 30Gbps to make a 1.2Tbps speed of system. The transmitting channel signal is passed through an optical fiber of link length $100 \mathrm{~km}$ along with DCF of length $8.37 \mathrm{~km}$. At the receiver section signal is received by 40 receivers and check the receiving signal strength on the basis of Eye diagram i.e., by the values of $Q$-factor, BER and eye opening of the signal provided by eye diagram analyzer. The system covers a distance of $100 \mathrm{~km}$ with good and favorable results on the basis of $Q$-factor and BER.
\end{abstract}

Keywords: DWDM, Tbps, DCF, Q-Factor, BER

\section{Introduction}

A dramatic increase in the data rate capacity of optical fiber communication system can thus be achieved by simultaneously transmitting of different optical signals to the same fiber from many different optical sources having particular values and proper spaced wavelengths. This technique is called Wavelength division multiplexing similar to Frequency division multiplexing which is used in microwave radio and satellite communication system. Increasing demand can be fulfilled by high speed and high capacity Dense wavelength division multiplexing networks. But high capacity DWDM networks need higher bandwidth (THz) which can be offered by medium that is optical fiber for better performances. DWDM improvement is a challenging technique in present time to meet this demand. High spectral efficiency in DWDM system can be achieved with an attractive bit rate of $>10 \mathrm{Gbps}$. In literature review, Demonstration of $96 \times 10 \mathrm{Gbps}$ DWDM system with $100 \mathrm{GHz}$ equal channel spacing for RZ and NRZ modulation format and compare the performances of system on the basis of Q-factor and eye closure. It is observed that NRZ provides good result amongst another modulation format and system using NRZ format covered a distance of $190 \mathrm{~km}$ with good and viable results [1]. Investigation of Long haul high capacity DWDM system optical networks using nonreturn to zero (NRZ) format by making impact of optical pulse shape on transmission performance and characteristics [2]. Demonstration of $64 \times 10 \mathrm{Gbps}$ and $96 \times 10 \mathrm{Gbps}$ DWDM system using RAMAN-EDFA hybrid optical amplifiers with NRZ, RZ and DPSK modulation formats and it was found that RZ is more adversely affected by nonlinearities as compared to NRZ and DPSK which are more affected by dispersion [3]. Investigation of $136 \times 10 \mathrm{Gbps}$ DWDM systems with channel spacing of $100 \mathrm{GHz}$ for NRZ and RZ formats and the results was found on the basis of Q-factor and BER. The system found that by using NRZ format Q-factor value is $20 \mathrm{~dB}$ and BER value is $10^{-25}$ better result than RZ format at $300 \mathrm{~km}$ having Q-factor 0.78dB [4]. Designing of 64 channels DWDM system having each channel data rate of $20 \mathrm{Gbps}$ with using Optimized modulation format to achieved the system capacity up to $1.28 \mathrm{Tbps}$. It was found that system having ultra high capacity of $1.28 \mathrm{Tbps}$ is expected to be more technically viable 
with use of only Optimized modulation format and this system is highly useful for high speed data processing computer networks [5]. Simulative analysis of 30 nodes ring network topology of DWDM system accommodating 90 users with using Erbium doped fiber amplifier and in the absence of dispersion compensating fiber. In this system total transmission distance achieved with each span is $1800 \mathrm{~km}$ having $70 \mathrm{~km}$ distance with each node and at $-22 \mathrm{dBm}$ input power to each user shows the acceptable Quality-factor and BER and also the network each user supported $25 \mathrm{Gbps}$ data rate [6]. Investigation of hybrid optical amplifiers with four modulation formats including Non return to zero (NRZ), Non return to zero raised cosine (NRZ-RC), Return to zero (RZ), Return to zero raised cosine (RZ-RC) and results finds that DWDM system with RZ and RZ-RC modulation format using RAMAN-EDFA hybrid optical amplifiers generates good results and transmission distance covered is 1512 and $1260 \mathrm{Km}$ respectively with Quality factor greater than $15 \mathrm{~dB}$ [7]. Studying of different techniques applied to the optical network to improve the data transmission rate in the network and concluded that by using different type of modulation schemes have effects on the performances of DWDM optical networks [8].Comparison of 40 Gbps DWDM system using various hybrid amplifiers with different modulation format and analyzed that EDFA-EDFA performed better than EDFA-SOA and also RZ format provides good result as compare with NRZ format [9].

\section{System Design}

To implement the performance of Tbps (Terabits per second) DWDM system having 40 channels, each channel having data rate of $30 \mathrm{Gbps}$ to make $40 \times 30 \mathrm{Gbps}$, that is $1.2 \mathrm{Tbps}$ high capacity or high speed optical network. A simulation set up for $1.2 \mathrm{Tbps}$ DWDM system is designed. This system set up consists of Transmitter section, and receiver section. The system results and performances are defined on the basis of BER, Qfactor and eye opening at 100km along with DCF of length $8.37 \mathrm{~km}$ having dispersion coefficient value that is $-80 \mathrm{ps} / \mathrm{nm}$, where system provides good and favourable results. The system set up block diagram as shown in Figure 1.

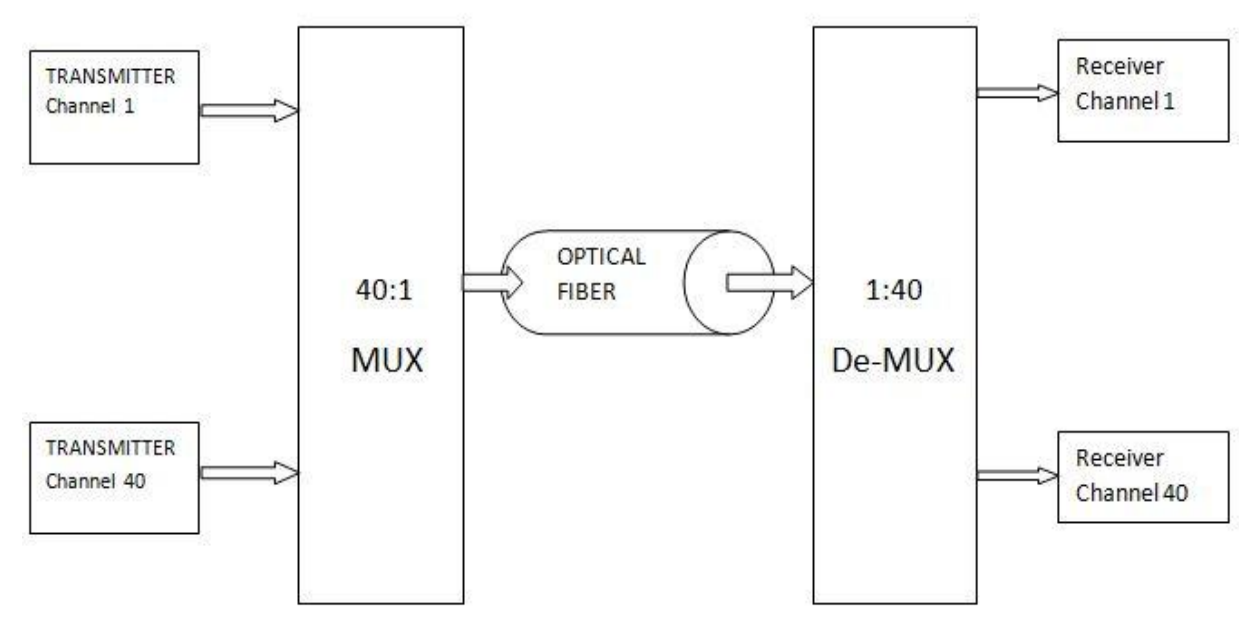

Figure 1. Block Diagram of System Set Up 
Table 1. System Set up Parameters of 1.2Tbps DWDM System

\begin{tabular}{|c|c|}
\hline System parameters & Values \\
\hline Number of channels & 40 \\
\hline Each channel data rate & $30 \mathrm{Gbps}$ \\
\hline Channels Frequency range & $193.1 \mathrm{THz}-197 \mathrm{THZ}$ \\
\hline Channel Spacing & $0.8 \mathrm{~nm}$ \\
\hline Attenuation & $0.2 \mathrm{~dB} / \mathrm{km}$ \\
\hline Dispersion of SMF & $16.75 \mathrm{ps} / \mathrm{nm} \cdot \mathrm{km}$ \\
\hline DCF length & $8.37 \mathrm{~km}$ \\
\hline Total link length & $100 \mathrm{~km}$ \\
\hline Modulation format & RZ \\
\hline Filter & Optical Bessel filter \\
\hline
\end{tabular}

\subsection{Transmitter Section}

Transmitter section consist of 40 channel transmitter each of them operating at their own frequency that is from $193.1 \mathrm{THz}$ to $197 \mathrm{THz}$ with equal channel spacing of $0.8 \mathrm{~nm}$, at a $30 \mathrm{Gbps}$ bit rate respectively for each channel. At transmitter section there is present 40 transmitter block, each block consist of pseudo random sequence as a data source having set their value of data rate is $30 \mathrm{Gbps}$ followed by a RZ coder for coding data into RZ form and Continuous wave laser as an optical source and a Modulator (match zehnder) which modulated the electrical signal from data source with optical signal from continuous wave laser to form an optical signal. Then these 40 optical signals are multiplexed by 40:1 multiplexer then transmitted into optical fiber having length $100 \mathrm{~km}$ along with DCF of length $8.37 \mathrm{~km}$. The transmitter block diagram of system set up as shown in Figure 2.

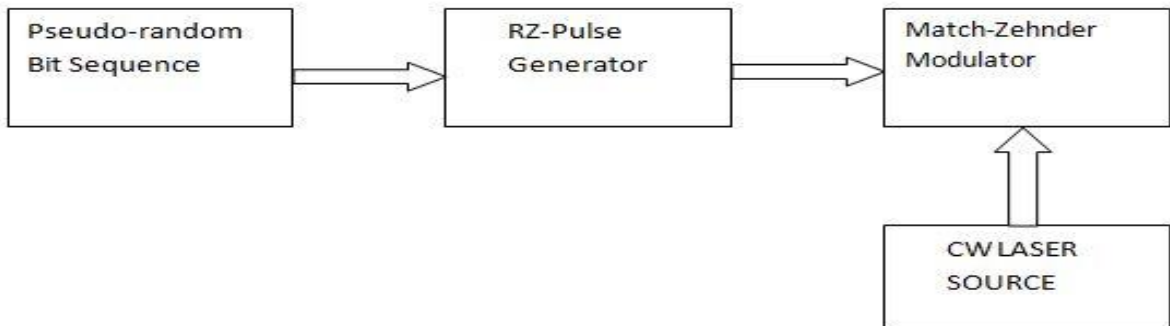

Figure 2. Transmitter Block Diagram of System Set up 


\subsection{Receiver Section}

At the receiver section there is present 40 receivers' block which demodulates the signal coming from optical fiber. The optical signal coming from fiber is first demultiplexed by 1:40 de-multiplexer, then passed through each receiver block for each channel. Each receiver block consists of PIN photodiode as a photo detector, which converts the optical signal into electrical signal and after photo detector there is present low pass rectangular filter, which filter the signal. After filteration process signal is passed to $3 \mathrm{R}$ regenerator which regenerates the signal and produces the data into original form. Followed by $3 \mathrm{R}$ regenerator in receiver block there is present Eye diagram analyzer which analyzed the signal and provides the values of Q-factor, BER and eye opening. The system results are defined by observing the values of Q-factor and BER. The receiver section system set up diagram as shown in Figure 3.

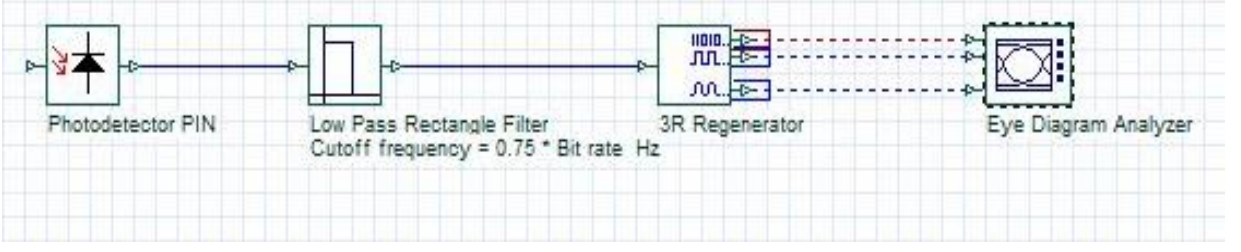

Figure 3. Receiver Set up for System

\section{Results and Discussion}

The 1.2Tbps (Terabits per second) DWDM system covers a distance of $100 \mathrm{~km}$ link length along with DCF of length $8.37 \mathrm{~km}$ for compensate dispersion and provides good and favourable result at this link length. In this system 40 transmitting channel, each of $30 \mathrm{Gbps}$ bit rate having frequencies range from $193.1 \mathrm{THz}$ to $197 \mathrm{THz}$, with equal channel spacing of $0.8 \mathrm{~nm}$ is transmitted by 40 transmitters block using 40:1 multiplexer, which multiplexed the signal into fiber of length $100 \mathrm{~km}$ along with DCF of length $8.37 \mathrm{~km}$. At the receiver section signal is de-multiplexed by 1:40 de-multiplexers into 40 receivers' block, which demodulated the signal into original form. The Eye diagram analyzer present in the receiver block gives the output of system by analysis the eye diagram of 40 receivers on the basis of Q-factor, BER and eye opening at $100 \mathrm{~km}$, where system generates good and viable results. The eye diagram of receiver channel 1 of $1.2 \mathrm{Tbps}$ DWDM system at 100km link length as shown in Figure 4.

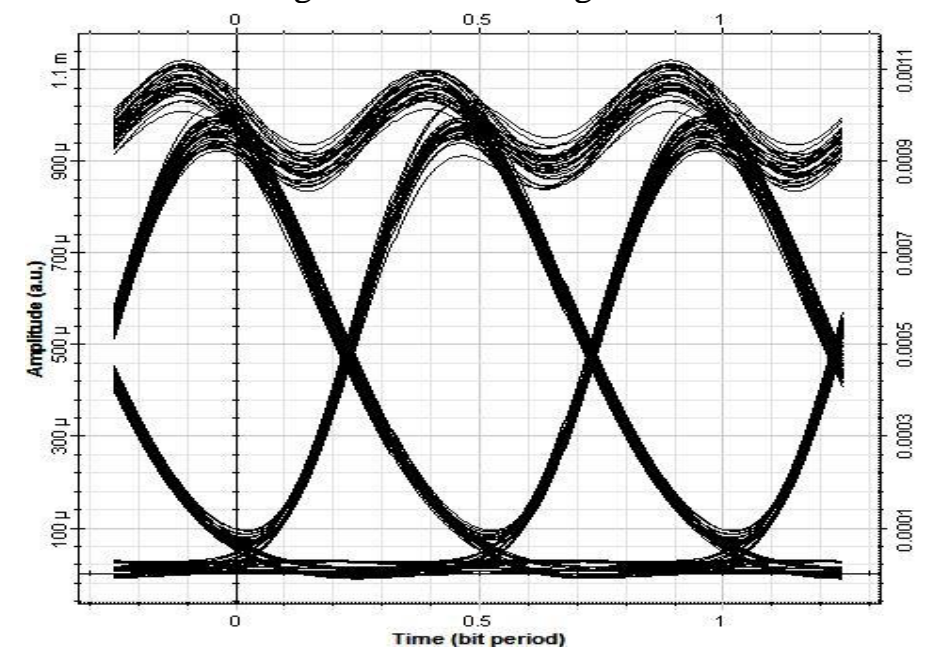

Figure 4. Eye Diagram of Receiver Channel 1 of DWDM System of 1.2Tbps 
The eye diagram of receiver channel 10 of $1.2 \mathrm{Tbps}$ DWDM system at $100 \mathrm{~km}$ link length as shown in Figure 5.

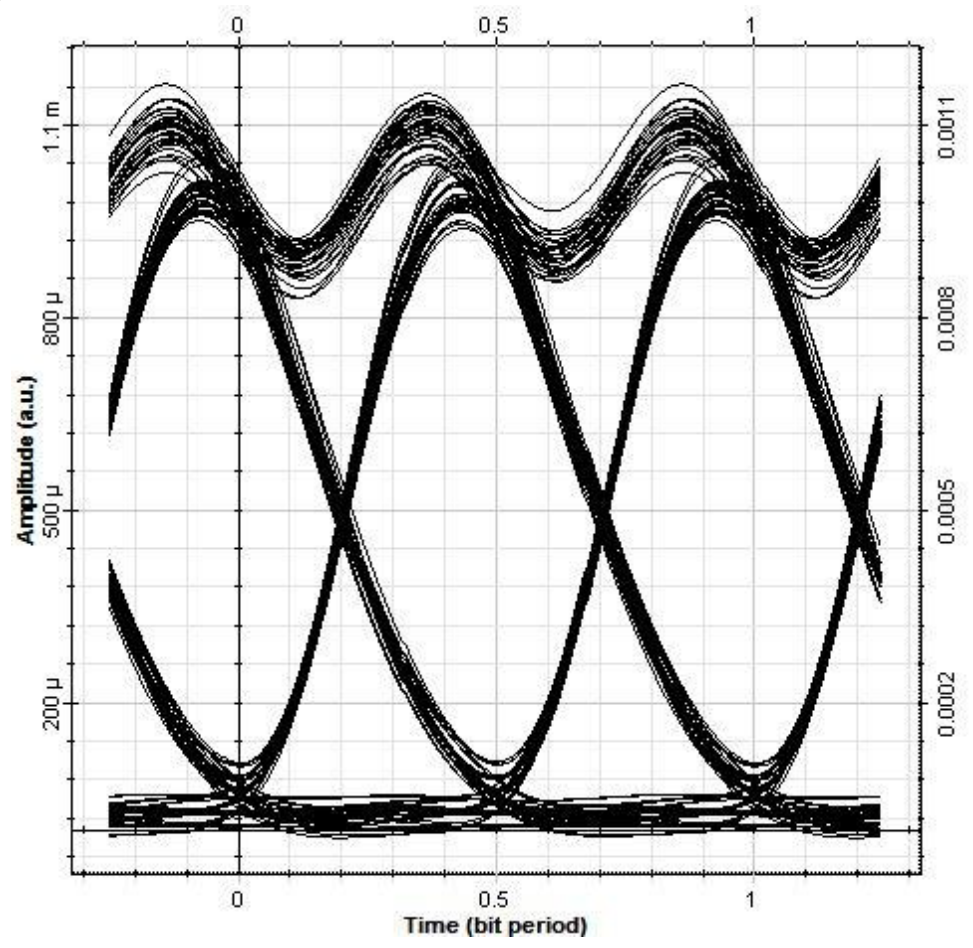

Figure 5. Eye diagram of Receiver Shannel 10 of DWDM System of 1.2Tbps

The eye diagram of receiver channel 20 of $1.2 \mathrm{Tbps}$ DWDM system at $100 \mathrm{~km}$ link length as shown in Figure 6.

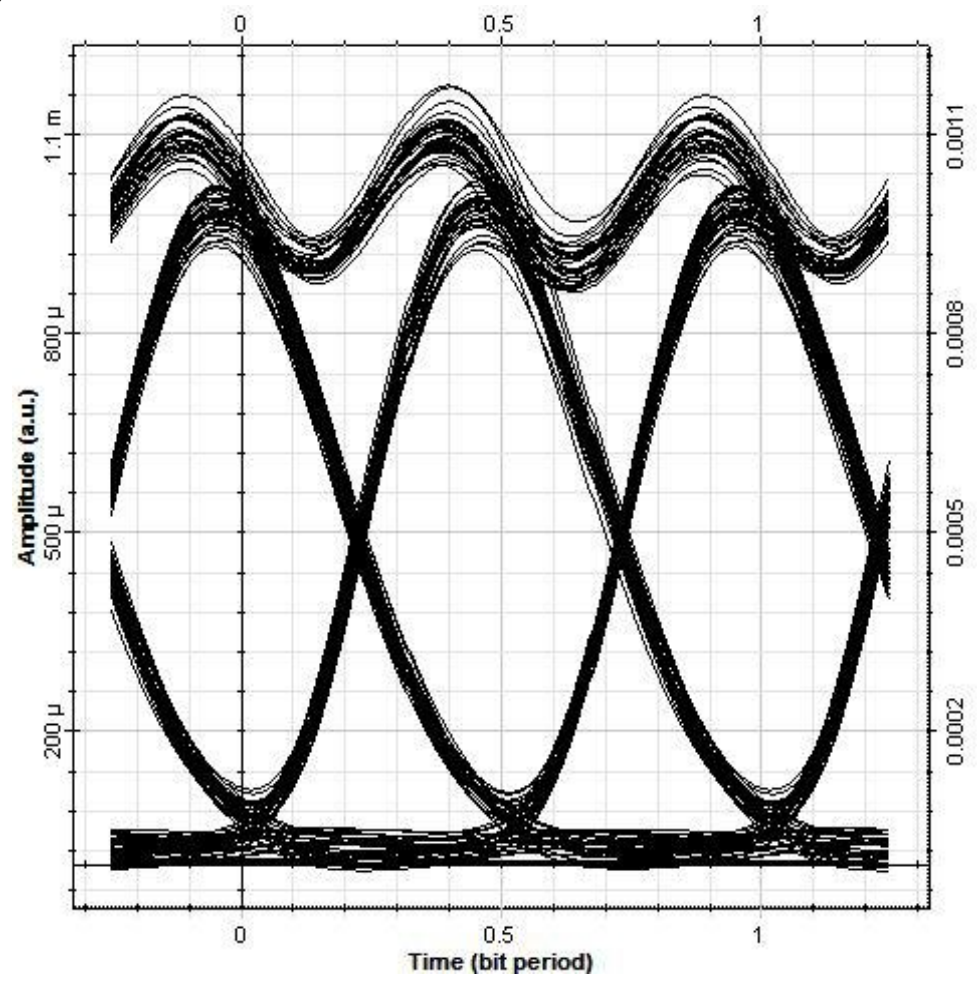

Figure 6. Eye Diagram of Receiver Channel 20 of DWDM System of 1.2Tbps 
The eye diagram of receiver channel 30 of $1.2 \mathrm{Tbps}$ DWDM system at $100 \mathrm{~km}$ link length as shown in Figure 7.

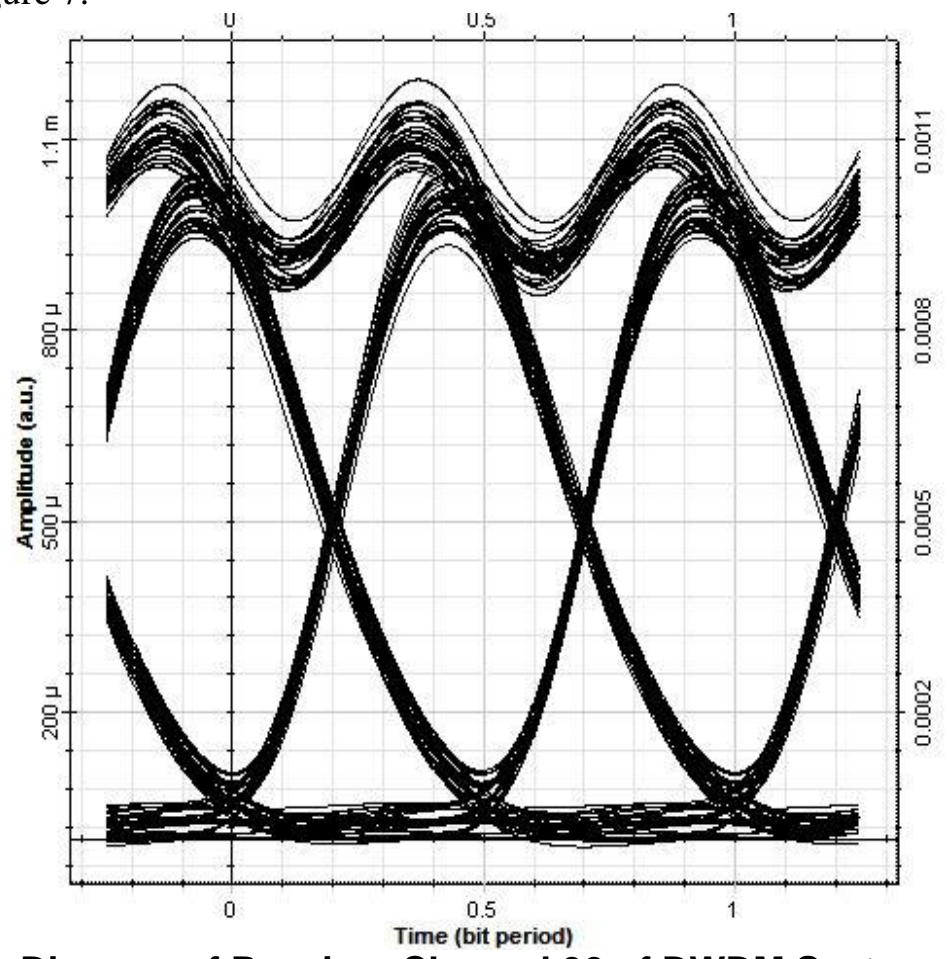

Figure 7. Eye Diagram of Receiver Channel 30 of DWDM System of 1.2Tbps

The eye diagram of receiver channel 40 of $1.2 \mathrm{Tbps}$ DWDM system at $100 \mathrm{~km}$ link length as shown in Figure 8.

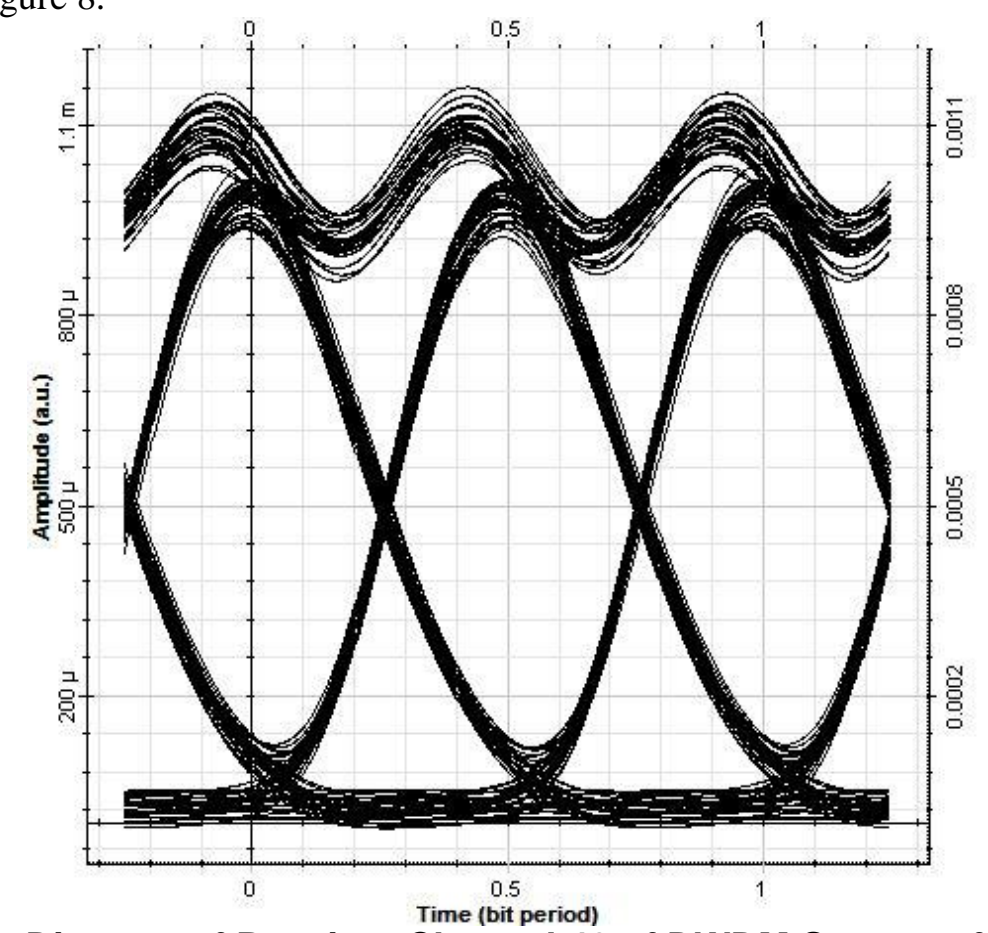

Figure 8. Eye Diagram of Receiver Channel 40 of DWDM System of 1.2Tbps 
Table 2. The Values of Q-factor and BER of Different Receiver Channels of System at $100 \mathrm{~km}$ Link Length of 1.2 Tbps DWDM System

\begin{tabular}{|c|c|c|}
\hline Receivers & Q-Factor & BER \\
\hline Receiver 1 & 17.27 & $10^{-67}$ \\
\hline Receiver 10 & 16.016 & $10^{-58}$ \\
\hline Receiver 20 & 15.06 & $10^{-51}$ \\
\hline Receiver 30 & 14.86 & $10^{-50}$ \\
\hline Receiver 40 & 14.34 & $10^{-47}$ \\
\hline
\end{tabular}

The results of DWDM system having Tbps capacity is analysed graphically by plotting Q-factor values of different receiver channels at 100, $200 \mathrm{~km}$ link length as shown in Figure 9. Graph shows that system generates good, acceptable results at $100 \mathrm{~km}$, having Q-factor values of all the receiver channels are greater than $14 \mathrm{~dB}$. But at $200 \mathrm{~km}$ system performance degrades because the valuesof Q-factor of all channels are reduces i. e. equal to between 6 and $7 \mathrm{~dB}$. From this it was found that system is good for short distance communication.

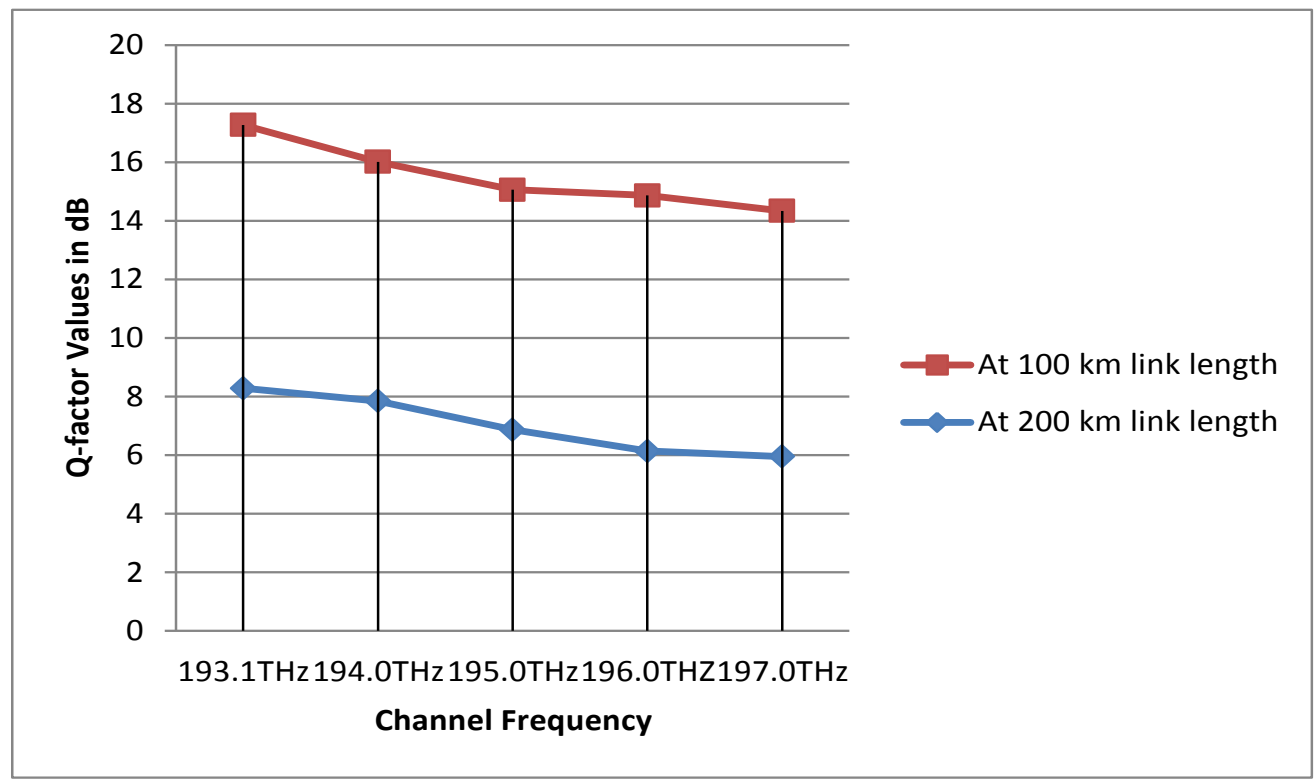




\section{Table 3. Comparison of Different Receiver Channels Q-factor Values at Different km}

\begin{tabular}{|c|c|c|}
\hline Receiver Channel & Q-factor at 100 $\mathbf{~ k m}$ & Q-factor at 100 $\mathbf{~ k m}$ \\
\hline $193.1 \mathrm{THz}$ & 17.27 & 8.28 \\
\hline $194.0 \mathrm{THz}$ & 16.016 & 7.85 \\
\hline $195.0 \mathrm{THz}$ & 15.06 & 6.87 \\
\hline $196.0 \mathrm{THz}$ & 14.86 & 6.14 \\
\hline $197.0 \mathrm{THz}$ & 14.34 & 5.95 \\
\hline
\end{tabular}

\section{Conclusion}

The performance of DWDM system with 40 channels having each channel bit rate of $30 \mathrm{Gbps}$ is defined on the terms of BER, Quality factor and eye opening at $100 \mathrm{~km}$ link length. It concludes that the system provides good result up to $100 \mathrm{~km}$ link length along with DCF of length $8.37 \mathrm{~km}$ and each channel Q-factor value is more than $14 \mathrm{~dB}$ at this link length and also system performance degrades at $200 \mathrm{~km}$. It is also concluded that system is operated at $1.2 \mathrm{Tbps}$ having high capacity and it was found that it is useful for high speed optical networks for short distance communication.

\section{Acknowledgments}

The author wishes to thanks Ms. Harmandar Kaur for the guidance provided in this research work.

\section{References}

[1] P. Singh, N. Singh, A. Singh, "Investigation of DWDM System for Different Modulation Formats", IOSR Journal of Engineering, Vol. 2, Issue 1, (2012), pp. 111-117.

[2] Y. J. Wen, A. Nirmalathas, "Impact of optical pulse shape on the performance of Long-Haul high capacity DWDM systems", Optics Communications 234, (2004), pp. 217-227.

[3] S. Singh, R. S. Kaler, "Performance evaluation of $64 \times 10$ Gbps and $96 \times 10$ Gbps DWDM system with hybrid optical amplifier for different modulation formats", Optik 123, (2012), pp. 2199-2203.

[4] P. Singh, N. S. Grewal, "NRZ versus RZ in 136 x 10 Gbps DWDM Systems", International Journal of Scientific \& Engineering Research, Volume 3, Issue 7, (2012).

[5] B. Patnaik, P. K. Sahu, "Ultra high capacity 1.28 Tbps DWDM system design and simulation using optimized modulation format", Optik 124, (2013), pp. 1567-1573.

[6] S. Singh, Raman, "Performance investigation on DWDM optical ring network to increase the capacity with acceptable performance", Optik 125, (2014), pp. 5750-5752.

[7] S. Singh, R. S. Kaler, "Investigation of hybrid optical amplifiers with different modulation formats for DWDM optical communication system", Optik 124, (2013), pp. 2131-2134.

[8] G. Garg, A. Singhal, "Investigation of Various Throughput Improvement Techniques in DWDM Optical Networks", International Journal of Advanced Research in Computer Science and Software Engineering, Volume 2, Issue 11, (2012), pp. 66-70.

[9] D. S. Sekhon, Jyotsana, J. Malhotra, "Investigation of 40 Gbps DWDM optical system using hybrid optical amplifier with different modulation formats", International Journal of signal processing and pattern recognition Vol. 8, No. 5, (2015), pp. 43-48. 